\title{
Estrategias de regulación emocional materna con bebés en situaciones de estrés: el uso del canto materno*
}

\section{Maternal Emotional Regulation Strategies with Infants in Stressful Episodes: Using Maternal Singing}

\author{
Olga Alicia Carbonell Blanco ${ }^{a}$ \\ Pontificia Universidad Javeriana, Colombia \\ ORCID: http://orcid.org/0000-0001-7603-5187 \\ Laura Estefanía García RodríGuez \\ Pontificia Universidad Javeriana, Colombia \\ Universidad de Navarra, España \\ ORCID: http://orcid.org/0000-0001-6919-2432 \\ Milton Eduardo Bermúdez-Jaimes \\ Pontificia Universidad Javeriana, Colombia \\ ORCID: http://orcid.org/0000-0002-5049-7073
}

a Autora de correspondencia. Correo electrónico: carbonel@javeriana.edu.co

Para citar este artículo: Carbonell Blanco, O. A., García Rodríguez, L. E., \& Bermúdez-Jaimes, M. E. (2019). Estrategias de regulación emocional materna con bebés en situaciones de estrés: el uso del canto materno. Universitas Psychologica, 18(5), 1-15. https:// doi.org/10.11144/Javeriana.upsy18-5.erem

\section{RESUMEN}

El propósito de este estudio fue caracterizar las estrategias regulatorias empleadas por madres en la interacción con sus bebés durante el primer año de vida en las situaciones de estrés que ocurrían en el contexto natural (hogar) de cuidado diario. Así mismo, determinar si el canto materno cumplía una función regulatoria de los estados fisiológicos y emocionales del bebé. Se utilizó un diseño de investigación no experimental de tipo transversal, con una muestra no probabilística por conveniencia de 32 díadas madre-bebé, que pertenecían a estratos socioeconómicos bajos. Los hallazgos de este estudio permitieron establecer dos patrones de cuidado materno: Patrón Regulatorio Contingente y Patrón Regulatorio Poco/No Contingente. También, se pudo identificar que el canto materno cumple, entre otras, una función regulatoria en situaciones de estrés en las rutinas de cuidado cotidiano.

\section{Palabras clave}

regulación emocional; canto materno; estrés en la crianza; relaciones madre-hijo; bebés.

\section{ABSTRACT}

The purpose of the study was to characterize maternal regulation strategies during mother-infant interactions at home in stressful episodes at everyday caregiving activities. Also, to identify if maternal singing accomplished the role of a physiological and emotional regulatory strategy in care routines. The methodological design was non-experimental, with a cross-sectional research method. The sample of 32 dyads (motherinfant) was recruited with a non-probabilistic method. The participants came from low-income socioeconomic status. Two patterns of maternal caregiving were found: Contingent Regulatory Pattern, and NonContingent Regulatory Pattern. As well, the maternal singing was identified to play different functions, among them, a regulatory one in stressful episodes during caregiving routines. 
Keywords

emotional regulation; maternal singing; parenting stress; motherchild relationships; infants.

Considerando que existen condiciones de vida inequitativas para una proporción significativa de niños y niñas en Latinoamerica (Unicef, 2009), es necesario pensar las problemáticas del desarrollo infantil que emergen en sus contextos relacionales de cuidado. Ello implica considerar el papel que juegan los adultos cuidadores, y en especial la familia, pues como señala el informe mundial sobre la infancia (Unicef, 2009), estos son los encargados de garantizar un "entorno protector" que permita el bienestar y el cumplimiento de los derechos de los niños y las niñas. Este interés global por fortalecer los ambientes infantiles está contemplado en la meta 4.2 de los Objetivos de Desarrollo Sostenible (Naciones Unidas, 2015), la cual señala que todas las niñas y los niños deben tener un desarrollo temprano de calidad, recibir cuidados y educación inicial preparatoria para la educación primaria. Es decir, se busca promover un desarrollo saludable y de bienestar psicosocial para la primera infancia.

El hincapié en la mejora de estas condiciones parte de la comprensión de los primeros años de vida como un periodo crucial, en el cual las experiencias que tienen lugar impactan significativamente en el desarrollo del cerebro, dada la masiva producción de conexiones sinápticas (Nelson, Furtado, Fox, \& Zeanah, 2009; Satchwell-Hirst, 2018). A esto se añade el rol que desempeñan los estresores en los ambientes de crianza caracterizados por la pobreza y desigualdad, los cuales pueden afectar negativamente la calidad del cuidado de los niños desde edades muy tempranas y, en consecuencia, incidir negativamente en el posterior desarrollo a nivel cognitivo, afectivo, social y emocional (Hackman, Gallop, Evans, \& Farah, 2015; Lipina \& Segretin, 2015; Winston \& Chicot, 2016). De ahí que sea relevante investigar sobre las relaciones de cuidado, especialmente en el primer año de vida.
Desde una perspectiva ecológica transaccional del desarrollo humano, el fracaso de cualquier ambiente inmediato (como es el microsistema familiar) a la hora de satisfacer las necesidades básicas de los niños, como ocurre en condiciones de pobreza y vulnerabilidad psicosocial, termina por afectar su desarrollo intraindividual desde el inicio de la vida (Cicchetti \& Valentino, 2006). Existen, por tanto, rangos de condiciones específicas de especie para elicitar procesos normativos del desarrollo. En el caso de los bebés, estos requieren cuidadores protectores y sensibles, y un grupo social amplio que apoye la socialización.

Estudios contemporáneos muestran que los procesos autorregulatorios y su relación con las prácticas de cuidado y crianza en la primera infancia - especialmente en el primer año de vida- requieren una aproximación desde un modelo biocomportamental de la sincronía cuidador-bebé (Feldman, 2012). Se sabe que los periodos iniciales de la vida son considerados "periodos sensibles" del desarrollo socioemocional (Perry, Blair, \& Sullivan, 2017), pues, desde una perspectiva neurobiológica, se dan diversos cambios y reorganizaciones bio-comportamentales, inscritos dentro de una relación madre/padre-bebé co-regulada y sincrónica, que parecen jugar un papel decisivo en los procesos y trayectorias del desarrollo cognitivo y socioemocional (Feldman \& Eidelman, 2009; Feldman, Gordon, Schneiderman, Weisman, \& Zagoory-Sharon, 2010).

Los estudios actuales en el campo de las neurociencias y el desarrollo infantil muestran que el desarrollo de las estructuras cerebrales mas importantes para la expresión y regulación emocional de las personas a lo largo de la vida están asociadas con la calidad y sensibilidad del cuidado que los niños reciban de sus padres o cuidadores familiares principales en la infancia, ya que los procesos co-regulatorios, que estos proveen a través de la relación, son los que permiten el desarrollo de dichas estructuras, que a su vez moldean el desarrollo emocional (Perry et al., 2017). 
En el mismo sentido, Sroufe (2016) señala que para la adaptación efectiva de las personas se necesitan condiciones de contexto, especialmente el relacional, que permitan el logro de determinadas tareas del desarrollo. En el caso de los bebés, las tareas principales del desarrollo son la formación de una relación de apego segura, el inicio de la reciprocidad, la regulación diádica del afecto y el equilibrio entre conductas de apego y exploración de su ambiente físico y social.

La evidencia aportada por la investigación en teoría del apego ha mostrado que la motivación de los niños pequeños por la exploración depende de la regulación de sus estados internos y excitaciones fisiológicas, así como de sus procesos cognitivos, afectivos y comportamentales. No obstante, debido al nivel de desarrollo neurológico del bebé en sus primeros años, que este logre procesos de regulación efectiva depende de un sistema de cuidado que es clave a la hora de apoyar los intentos del bebé de modular estados emocionales (Ainsworth, Bell, \& Stayton, 1974; Calkins \& Hill, 2007; Crespo, Trentacosta, Aikins, \& Wargo-Aikins, 2017; King, Humphreys, \& Gotlib, 2019; Miller \& Commons, 2010; Perry et al., 2017; Sroufe, Egeland, Carlson, \& Collins, 2005; Tronick \& DiCorcia, 2015). Las experiencias emocionales intensas, ya sean negativas o positivas, desbordan la capacidad del bebé para autorregularse y, por tanto, se requiere la co-regulación por parte del cuidador (Cooper, Hoffman, Powell, \& Marvin, 2007; Sroufe, Szteren, \& Causadias, 2014). Es decir, es un proceso de adaptación diádica, a través de una co-regulación guiada por el adulto que cuida (Sroufe et al., 2005).

Este proceso conlleva un cambio progresivo de co-regulación diádica hacia la regulación autónoma y efectiva, que se traduce en el desarrollo de estrategias conductuales y cognitivas para hacer frente a las experiencias emocionalmente excitantes. Esto se va convirtiendo en un sistema emocional regulatorio del self (el sí mismo) (Sroufe et al., 2005; Sroufe et al., 2014), que tiene enormes implicaciones para el desarrollo de la capacidad de autorregulación y de adaptación social del niño en momentos posteriores del desarrollo (Coppola, Ponzetti, Aureli, \& Vaughn, 2016).

Los supuestos teóricos y la evidencia empírica muestran la sincronía que se da en las interacciones entre los adultos cuidadores y los niños pequeños (Feldman, 2012). Incluso, madres sin experiencia con sus bebés recién nacidos son capaces de regularse mutuamente, entender sus señales comunicativas y responder de manera contingente a las mutuas acciones (Retana Franco \& Sanchéz-Aragón, 2010; Tronick \& DiCorcia, 2015). Una interacción sincronizada en la díada comprende la expresión recíproca de diversos estados afectivos, y los intercambios son en general positivos, lo cual provee al niño de un contexto seguro y de apoyo en el cual manifestar sus emociones y aprender a regularlas de forma efectiva (De Rubeis \& Granic, 2012; Pratt, Singer, KanatMaymon, \& Feldman, 2015), lo que a futuro previene problemas de comportamiento (ImBolter, Anam, \& Cohen, 2015), especialmente en poblaciones de alto riesgo y en vulnerabilidad psicosocial (Causadias, Salvatore, \& Sroufe, 2012).

Otros estudios han mostrado que la regulación emocional de los bebés también está asociada a la disponibilidad emocional materna (Thomas, Letourneau, Campbell, Tomfohr-Madsen, \& Giesbrecht, 2017): a mayor hostilidad manifiesta de la madre en las interacciones, mayor dificultad del bebé para regular emociones negativas durante y después de situaciones estresoras. En sentido contrario, cuando la madre emplea un estilo sensible y un cuidado estructurador del ambiente, no intrusivo y no intrusivo ni hostil, facilita la regulación emocional y comportamental del bebé (Little \& Carter, 2005).

Los comportamientos que tienen como meta regular y mantener la experiencia emocional han sido llamados estrategias de regulación emocional (Diener, Mangelsdorf, McHale, \& Frosch, 2002). Observaciones pioneras de bebés a finales de la década del 50 y 60, reportadas por Bowlby (1993), muestran que los estados emocionales negativos del bebé, como el llanto, eran calmados de manera eficaz, cuando las cuidadoras utilizaban diversas 
estrategias: acunarlo, proporcionarle succión sin fines alimenticios y hablarle. Estudios recientes han observado que el canto animado (o canciones multimodales) de la madre a sus hijos mientras estos lloran, tiene un mejor efecto regulador, que se prolonga más en el tiempo y disminuye mejor los estados de excitación, en comparación al habla materna (Trehub, Ghazban, \& Corbeil, 2015).

Diversos autores señalan que las canciones de cuna se utilizan en las distintas culturas en la interacción personal e íntima de los niños con los adultos cuidadores como rituales preparatorios del sueño o para calmar estados de excitación emocional y fisiológica de los bebés (Altmann de Litvan et al., 2001; Bauer et al., 2007; Baker \& Mackinlay, 2006; Bargiel, 2004; L'Etoile, 2006; Trehub et al., 2015; Vrushali \& Shefali, 2011). Algunos estudios han hallado asociaciones entre el canto de la madre con una reducción en los niveles de cortisol del bebé (Shenfield, Trehub, \& Nakata, 2003). Por tanto, la canción de cuna no es únicamente una herramienta para la socialización dentro de la propia cultura, sino que parece ser un recurso útil para regular los estados emocionales y fisiológicos del bebé (Bargiel, 2004). Así mismo, al involucrar el contacto físico, mediante el mecer y acunar, el canto contribuye en la construcción del vínculo afectivo entre la madre y su hijo (Idoyaga, 2008; Vrushali \& Shefali, 2011).

La mayoría de los estudios sobre los procesos de regulación emocional en la primera infancia se focalizan en las estrategias que los niños van adquiriendo progresivamente para autorregularse, y el papel que juegan las madres y/ o padres para favorecer este proceso, en función de las relaciones de apego que establecen (Beebe et al., 2010; Coppola et al., 2016; Diener et al., 2002; Qu, Leerkes, \& King, 2016; Riva Crugnola et al., 2011; Sherman, Stupica, Dykas, RamosMarcuse, \& Cassidy, 2013) y/o del nivel de sensibilidad del cuidador (Leerkes, Crockenberg, \& Burrous, 2004; Leerkes, Blankson, \& O’Brien, 2009). No obstante, existe escasa evidencia empírica sobre los recursos comportamentales que de manera espontánea usan las madres para apoyar los procesos co-regulatorios madre- bebé, especialmente, en el primer año de vida, y específicamente en contexto natural, así como del papel que juega el canto materno en las rutinas de cuidado como una estrategia de crianza.

Por lo anterior, las preguntas indagadas fueron: 1) iqué tipos de estrategias regulatorias usan las madres con sus bebés en sus rutinas de cuidado diario en situaciones de estrés, y entre estas utilizan la canción de cuna?, y 2) iqué funciones cumplen estas estrategias para ayudar a regular emocionalmente al bebé?

\section{Método}

Diseño

Se utilizó un diseño investigación no experimental de tipo transversal (Hernández, Fernández, \& Baptista, 2010), cuyo propósito fue explorar un campo de conocimiento sobre las relaciones de cuidado madre-bebé, relativamente nuevo en nuestro contexto.

\section{Participantes}

Se seleccionó una muestra no probabilística por conveniencia de 32 madres de la ciudad de Bogotá, Colombia. Las díadas pertenecían a los estratos socioeconómicos bajos 1 y 2 (excepto una del nivel 3) sobre una escala de 6 puntos (Departamento Administrativo de Planeación Distrital, 2005). Las edades promedio fueron $M$ $=27.6$ años, $D E=5$ en un rango entre 21 y 40 años. Los bebés tenían de 7-12 meses de edad (M $=8.9$ meses, $D E=1.2)$, de los cuales eran 20 niñas y 12 niños. Respecto a ingresos, la mayoría de las familias ganaba entre 1 y 2 salarios mínimos legales $(78.1 \%)$. En cuanto al nivel educativo de las madres, la mayoría tenía un nivel de formación de secundaria incompleta o completa (56.26\%), seguido por un nivel formativo técnico incompleto o completo $(28.13 \%)$. Con relación al estado civil de las madres, se hallaban en unión libre el $46.9 \%$, solteras el $37.5 \%$, casadas el 12.5 $\%$ y divorciadas el $3.1 \%$. 


\section{Instrumentos y procedimientos}

La medición de las variables de interés del estudio se realizó en dos sesiones, contexto natural (hogar) y contexto experimental (laboratorio). En primer lugar, se realizó una visita domiciliaria de 2.5 horas, con dos observadores entrenados que participaron con las díadas madre-bebé durante las rutinas de cuidado, focalizándose en los episodios de estrés. Las rutinas fueron filmadas para un análisis posterior, que permitiera identificar las estrategias regulatorias maternas durante las interacciones madre-bebé, y establecer cuáles madres cantaban a sus bebés. Adicionalmente, las díadas participaron en una visita de laboratorio durante una hora, donde fueron observadas en una sesión de juego libre de 5 minutos con el fin de identificar en este contexto las madres que cantaban a sus bebés.

Durante la visita domiciliaria se aplicó una encuesta para recolectar información concerniente a condiciones socioeconómicas, salud física y emocional de la díada, redes de apoyo, y eventos estresantes del entorno familiar y de crianza. Para recolectar la información referente a la variable Estrategias de Regulación Materna, se emplearon dos métodos. El primero fue realizar registros en videos de los comportamientos de las madres durante las interacciones con su bebé, específicamente aquellos empleados de manera intencional para calmar la emocionalidad negativa de sus bebés durante las situaciones de estrés en las rutinas de cuidado diario. El segundo medio de recolección fue el Cuestionario de Prácticas de Cuidado I de pregunta abierta, construido por los investigadores, y validado a través de 4 jueces expertos en la temática, considerando los siguientes criterios: redacción, coherencia, pertinencia y ubicación de cada uno de los reactivos que componen el instrumento, que indagó sobre las experiencias de cuidado diario, y especialmente sobre las estrategias regulatorias empleadas por las madres para facilitar el desarrollo de las rutinas de cuidado de su bebé durante los momentos estrés. En este cuestionario se solicitaba a las madres identificar y jerarquizar las estrategias regulatorias utilizadas con sus bebés para cada una de las diferentes rutinas (alimentación, cambio de pañal, baño, vestir y desvestir, juego y preparación para el sueño).

Para evaluar la variable Uso de la Canción de Cuna, durante las visitas domiciliaria y experimental, se observó si de manera espontánea las madres hacían uso de la canción de cuna durante las interacciones con sus bebés. Para aquellas madres que cantaron en por lo menos uno de los dos contextos, se aplicó el Cuestionario de Prácticas de Cuidado II (Altmann de Litvan et al., 2001). Esto, con el fin de explorar y profundizar sobre las funciones que cumplía la canción de cuna, las situaciones en las cuales era empleada, los tipos de canciones, así como los sentimientos generados en las madres y su percepción sobre las emociones que el canto generaba en sus bebés.

Con el propósito de identificar y caracterizar las estrategias regulatorias maternas, los registros en video se sometieron a un análisis posterior con el Software Observer XT 10.5, a través del cual se codificaron las conductas que las madres usaban para regular a sus bebés. En el aparte de resultados se explican los procedimientos seguidos para garantizar la confiabilidad de la información recolectada a través de los videos. Los Cuestionarios de Prácticas de Cuidado I y II fueron codificados a través del Sistema software Nvivo 10. El procesamiento fue realizado por tres codificadores, y para garantizar la confiabilidad de la codificación, se usó el siguiente procedimiento: tres combinatorias posibles de parejas codificadoras que se repetían cada tres cuestionarios, y procesaron de manera independiente las preguntas, que obtuvieron un acuerdo de calificación por encima del $80 \%$.

\section{Resultados}

A continuación, se presentan los análisis de resultados de las Estrategias Regulatorias Maternas. El procedimiento seguido para analizarlas partió de un estudio piloto (González Ramírez, 2011), realizado para indagar sobre 
los comportamientos que de manera intencional empleaban las madres para regular a sus bebés (entre 10-15 meses de edad) en situaciones de estrés durante rutinas de cuidado diario. Las conductas maternas observadas se utilizaron como preliminares para establecer un primer repertorio comportamental. No obstante, debido a que la muestra de niños del presente estudio estaba en un rango de edad menor que el estudio piloto, se consideró necesario revisar la totalidad de los videos de las visitas domiciliarias, para establecer si los comportamientos identificados en el piloto se correspondían con los que presentaban las madres y los bebés del presente estudio. Luego de la revisión exhaustiva de los videos, se conservó la mayoría de las conductas maternas y de los bebés. También, se incorporaron algunas nuevas observadas, y se eliminaron aquellas que no aplicaban al rango de edad de los bebés del presente estudio.

Se codificaron los videos para las 32 díadas, mediante el software Observer XT 10.5, donde se diferenciaron las codificaciones por rutinas y se seleccionó el episodio de estrés más significativo en cada una de ellas. El proceso de codificación fue realizado por dos observadores, quienes inicialmente se encargaron de procesar las conductas maternas y de los bebés. Cada observador se encargó de procesar el $50 \%$ de los videos. El observador 1 codificó los 32 episodios de aseo (baño, cambio de pañal, vestir y desvestir) más los 16 primeros episodios de juego y preparación al sueño. El observador 2 codificó los 32 episodios de alimentación más los otros 16 restantes de juego y preparación para el sueño. A través de este procesamiento, primero se establecieron frecuencias de conductas por parte de la madre y del bebé. Las conductas de mayor ocurrencia por parte de ambos miembros durante los episodios de estrés identificadas fueron organizadas en orden de jerarquía.

El procedimiento anterior permitió identificar las conductas de las madres y los bebés de forma aislada, por fuera de la interacción y sin discriminarlas por rutina. Seguido a esto, se sometió la información a un segundo análisis para recuperar las secuencias de interacción entre la díada, y establecer las estrategias regulatorias empleadas por las madres y su efectividad. Así mismo, se identificaron los comportamientos maternos más frecuentes, aquellos que eran empleados de manera general o los que se diferenciaban por rutina.

Para controlar el sesgo de observador en esta segunda fase de análisis de secuencias interactivas madre-bebé, se utilizó un tercer observador, y se rotaron y se distribuyeron las codificaciones de la siguiente forma: el observador 1 esta vez codificó las 32 díadas durante la rutina de alimentación, el observador 2 se encargó de codificar las 32 díadas en los episodios de aseo, y el observador 3 codificó las rutinas de juego y preparación para el sueño para las 32 díadas, donde se halló una concordancia mayor al 80 \% con relación a la codificación anterior.

Al analizar y organizar la información recolectada por observación directa y por reporte materno (cuestionario), se identificaron dos tipos de patrones de cuidado materno, con sus respectivos comportamientos, los cuales fueron considerados como diferentes tipos de estrategias. El primer patrón de cuidado se denominó Regulación Materna Contingente, que se definió al tener en cuenta aquellas conductas de la madre que involucraban disponibilidad física, psicológica y emocional ante las señales comunicativas del bebé, emociones positivas mutuas, intentos de la madre de generar satisfacción y bienestar a su hijo, al que consideraba un interlocutor válido y activo. Este tipo de estrategia también implicaba eficacia en términos de la respuesta del bebé (reducción y/o eliminación de las señales de estrés y emergencia de conductas de satisfacción y tranquilidad). En este patrón de cuidado, las madres manifestaban conciencia sobre la intencionalidad y las necesidades comunicativas del bebé, y buscaban responder a estas apropiadamente.

El segundo patrón de cuidado se denominó Regulación Materna Poco/No Contingente, pues agrupó un conjunto de estrategias de la madre, caracterizadas por la evitación de la proximidad física hacia el bebé, y la manifestación de poca disponibilidad psicológica y emocional para regular las emociones y estados negativos de su 
hijo. También comprende atribuciones negativas a los estados psicológicos e intencionalidades del bebé, así como altas expectativas sobre la capacidad del bebé para regularse por sí mismo. En este patrón regulatorio, las madres empleaban diversas conductas que intentaban reducir el estrés del bebé, sin embargo, en la mayoría de las ocasiones, no eran eficaces. Durante los episodios de estrés, ambos miembros de la díada se desregulaban emocional y comportamentalmente, donde las madres informaban que distanciarse del bebé las ayudaba a tranquilizarse y a reducir su propio estrés.

A continuación, se presentan los dos patrones de cuidado materno previamente descritos, con sus respectivas estrategias (comportamientos) y definiciones:

\section{Tabla 1}

Estrategias identificadas en la rutina de cuidado Regulación Materna Contingente

\begin{tabular}{|c|c|c|}
\hline Estrategias & Definición Comportamental & Frecuencia \\
\hline Hablarle & Regular y confortar al bebé por medio de verbalizaciones hacia este. & \\
\hline Contacto fisico & $\begin{array}{l}\text { Calmar al bebé mediante el contacto fisico. Por ejemplo: alzar, abrazar, } \\
\text { cargar, consentir, arrullar, acariciar, etc. Implican proximidad y } \\
\text { disponibilidad fisica. }\end{array}$ & 54 \\
\hline Dar alimento & $\begin{array}{l}\text { Proveer alimento al bebé como un medio para facilitar el tránsito al } \\
\text { sueño, cuando este expresa malestar durante la rutina de sueño. }\end{array}$ & 31 \\
\hline Distraer con objetos & $\begin{array}{l}\text { Proporcionar juguetes u otra clase de objetos para entretener al bebé, } \\
\text { con la intención de facilitar la rutina cuando esta se torna difícil. }\end{array}$ & 27 \\
\hline Jugar/Entretener & $\begin{array}{l}\text { Divertir y distraer con actividades o juegos al bebé de manera } \\
\text { intencional cuando la rutina se dificulta. }\end{array}$ & 21 \\
\hline Cantarle & $\begin{array}{l}\text { Cantar al bebé cuando expresa una emocionalidad negativa para } \\
\text { facilitar el desarrollo de la rutina. }\end{array}$ & 13 \\
\hline No obligar & $\begin{array}{l}\text { Capacidad para entender cuándo el bebé está satisfecho y no desea } \\
\text { comer más, respondiendo acorde al estado fisiológico de su bebé. }\end{array}$ & 10 \\
\hline $\begin{array}{l}\text { Ajustarse al ritmo y } \\
\text { estado del bebé }\end{array}$ & $\begin{array}{l}\text { Coordinarse en la interacción con el bebé en función de los ritmos y } \\
\text { estados que percibe en él. }\end{array}$ & 9 \\
\hline $\begin{array}{l}\text { Sustituir/complementar } \\
\text { con otro alimento }\end{array}$ & $\begin{array}{l}\text { No obligar al bebé a recibir más un alimento cuando no desea, sino } \\
\text { sustituirlo con otro que reciba mejor. }\end{array}$ & 8 \\
\hline $\begin{array}{l}\text { Estrategias cuando el } \\
\text { bebé está enfermo }\end{array}$ & $\begin{array}{l}\text { Poner mayor empeño en confortar el malestar del bebé cuando está } \\
\text { enfermo, empleando otras estrategias adicionales a las habituales. }\end{array}$ & 7 \\
\hline $\begin{array}{l}\text { Autorregulación de la } \\
\text { madre }\end{array}$ & $\begin{array}{l}\text { Regular en si misma aquellas señales de tensión emocional o estrés que } \\
\text { pueden dificultar el cuidado del bebé. Hay conciencia sobre las propias } \\
\text { emociones y la importancia de regularlas para poder contener las de su } \\
\text { hijo. }\end{array}$ & 6 \\
\hline Identificar necesidades & $\begin{array}{l}\text { Verificar si el malestar del bebé se debe a que alguna necesidad básica } \\
\text { no está cubierta. Ejemplo, comprobar si está sucio el pañal, o tiene } \\
\text { hambre. }\end{array}$ & 6 \\
\hline $\begin{array}{l}\text { Anticiparse y } \\
\text { complacer al bebé }\end{array}$ & $\begin{array}{l}\text { Se refiere a las conductas que despliega la madre para anticipar y evitar } \\
\text { aquellas situaciones que ella reconoce como desencadenantes del estrés } \\
\text { del bebé. }\end{array}$ & 5 \\
\hline Acompañar & $\begin{array}{l}\text { Permanecer cerca del bebé para que sienta su presencia y expresarle } \\
\text { disponibilidad fisica y emocional. Implica reconocer subjetividad del } \\
\text { bebé, y cómo su presencia y compañia son importantes para él. }\end{array}$ & 5 \\
\hline Insistir & $\begin{array}{l}\text { Insistir al bebé para que reciba comida, sin llegar a obligarlo, } \\
\text { respetando hasta el punto en que indique estar satisfecho. }\end{array}$ & 1 \\
\hline Dormirlo & $\begin{array}{l}\text { Acostar a dormir al bebé para luego desarrollar mejor la rutina de } \\
\text { alimentación. }\end{array}$ & 1 \\
\hline
\end{tabular}

\section{Tabla 2}

Estrategias identificadas de la rutina de cuidado Regulación Materna Poco/No Contingente

\begin{tabular}{|c|c|c|}
\hline Estrategias & Definición comportamental & Frecuenci \\
\hline $\begin{array}{l}\text { Autorregulación } \\
\text { del bebé }\end{array}$ & $\begin{array}{l}\text { Altas expectativas frente a la capacidad del bebé para calmarse solo } \\
\text { cuando llora. Hay distanciamiento y disminución significativa de la } \\
\text { proximidad y el contacto. Esta estrategia lleva al bebé a intensificar el } \\
\text { llanto de manera descontrolada y luego cesar por cansancio. }\end{array}$ & son \\
\hline Regañar & $\begin{array}{l}\text { Elevar el tono de la voz al dirigirse al bebé, para hacerle entender su } \\
\text { enojo o desaprobación por no obedecer. La madre impone su voluntad } \\
\text { sin considerar los estados emocionales y/o fisiológicos del bebé. }\end{array}$ & \\
\hline Obligar & $\begin{array}{l}\text { Continuar el desarrollo de la rutina hasta finalizarla, a pesar del } \\
\text { malestar y displacer del bebé, sin negociar o buscar alternativas que le } \\
\text { regulen. }\end{array}$ & \\
\hline $\begin{array}{l}\text { Estrategias } \\
\text { ineficaces }\end{array}$ & $\begin{array}{l}\text { Emplear el ensayo y el error para lograr regular a su bebé. A pesar de } \\
\text { usar diversos comportamientos, la madre no logra calmar al bebé de } \\
\text { manera pronta y adecuada. }\end{array}$ & \\
\hline $\begin{array}{l}\text { Amena } \\
\text { pegar }\end{array}$ & $\begin{array}{l}\text { Amenazar de manera verbal o gestual al bebé con castigarle } \\
\text { fisicamente, si no come o hace la actividad al ritmo que la madre desea. }\end{array}$ & \\
\hline
\end{tabular}

Como se observa en las Tablas 1 y 2 , las conductas maternas son consideradas estrategias, pues denotan intencionalidad para responder o reducir las señales de emocionalidad negativa o malestar del bebé.

\section{Análisis de la variable uso de la canción de cuna}

Los resultados mostraron que $56.25 \%$ de las madres cantaban a sus bebés, ya fuera en el contexto del hogar y/o del laboratorio, mientras que un $43.75 \%$ no cantó en ninguno de los dos contextos. Así mismo, se identificaron distintos tipos géneros de canciones usadas por las madres: canciones infantiles tradicionales y modernas multimodales (55.5\%), música bailable popular (31.5\%), otro tipo de música $(7.4 \%)$ y canciones de cuna $(5.5 \%)$.

A través de los análisis de las situaciones específicas observadas y reportadas, se pudo establecer que el canto materno en las interacciones con el bebé cumplía diferentes funciones en el cuidado de este.

\section{Tabla 3}

Funciones del canto materno en interacciones con el bebé

\begin{tabular}{lr}
\hline \multicolumn{1}{c}{ Funciones } & Frecuencia \\
\hline $\begin{array}{l}\text { Facilitar el desarrollo y cumplimiento de las rutinas de cuidado creando un } \\
\text { ambiente interesante para el bebé }\end{array}$ & $40.6 \%$ \\
$\begin{array}{l}\text { Entretener y divertir al bebé mediante el canto como una actividad lúdica, ya } \\
\text { sea a través de interacciones entre la díada madre-bebé o en compañía de otros } \\
\text { familiares cercanos }\end{array}$ & $37.5 \%$ \\
$\begin{array}{l}\text { Calmar al bebé en situaciones de estrés donde este manifiestaba emocionalidad } \\
\text { negativa, en este sentido el canto materno cumplía una función regulatoria }\end{array}$ & $31.3 \%$ \\
$\begin{array}{l}\text { Usar el canto como acompañamiento al bebé mientras la madre está ocupada en } \\
\text { tareas domésticas, de manera que este no se sintiera solo }\end{array}$ & $12.5 \%$ \\
\hline
\end{tabular}

\section{Discusión}

En este estudio se buscó identificar y caracterizar las estrategias regulatorias maternas en el primer año de vida del bebé, temática muy relevante conceptual y empíricamente en el dominio de cuidado infantil y las relaciones cuidador-bebé en edades muy tempranas.

$\mathrm{Al}$ analizar los comportamientos maternos que componen el Patrón Regulatorio Contingente, se aprecia que muchas de las conductas y actitudes involucraban la mutua expresión de diversos estados afectivos, y los intercambios eran caracterizados, en general, por emociones 
positivas y regulaciones, que generaban comportamientos coordinados entre ambos miembros de la díada. Esto se corresponde con los componentes que definen el funcionamiento contingente de un sistema diádico temprano a través de la reciprocidad, sintonización y coregulación (Feldman, 2012; Pratt et al., 2015; Tronick \& DiCorcia, 2015). Los hallazgos de este patrón de cuidado también apoyan los resultados de otras investigaciones que describen la sincronía que emerge en las interacciones entre los adultos cuidadores y los niños pequeños, donde ambos son capaces de coordinar sus acciones para mutuamente regularse y entender las señales comunicativas del otro (Little \& Carter, 2005; Retana Franco \& Sanchéz-Aragón, 2010). Igualmente, se observó que cuando los bebés manifestaban llanto o quejidos para expresar algún malestar físico o psicológico durante la interación, las madres modulaban su estado afectivo y su comportamiento con el del bebé, para regularlo emocionalmente, tal como se ha encontrado en otros estudios (Riva Crugnola et al., 2011). Asimismo, las madres usaban estrategias cuyo propósito era transformar el estado de estrés del bebé en uno no-estresante, donde exhibían conductas de reparación de los micro-desencuentros interactivos en afectos e intenciones de la díada (Tronick \& DiCorcia, 2015).

En este patrón regulatorio se observó que el contacto físico materno facilitaba los procesos de regulación fisiológica y comportamental de los bebés que, al igual que en otras investigaciones, que señalan que proveer contacto físico al bebé es contingente, ya que le ayuda a disminuir los niveles de estrés y de cortisol durante actividades de juego libre (Feldman, Singer, \& Zagoory, 2010), y propicia comportamientos autorregulatorios, que los organiza alrededor del afecto y la atención mutua (Jean, Stack, \& Arnold, 2014). Este tipo de experiencias de sintonización afectiva madre-bebé, según la evidencia empírica, le proveen a este un contexto seguro y de apoyo, en el que puede manifestar sus emociones y aprender a regularlas efectivamente (De Rubeis \& Granic, 2012).
Más aun, para que el bebé logre procesos autónomos de regulación efectiva, es decir, desarrolle progresivamente la capacidad de autorregularse, primero depende de un sistema de cuidado que apoye ese proceso y actúe como un agente co-regulador de las emociones que tienen el potencial para desbordarlo (Cooper et al., 2007; Sroufe, 2016).

Adicionalmente, se evidenció que el Patrón Regulatorio Contingente engloba elementos que son congruentes con el constructo de la sensibilidad materna, tal como fue definido conceptual y operacionalmente, por Ainsworth et al. (1974), observados también en otros estudios sobre los procesos de regulación emocional con bebés y sus asociaciones con los comportamientos de sensibilidad materna o parental (Leerkes et al., 2004). Estos elementos se refieren a los comportamientos donde las madres a) manifiestan disponibilidad física, emocional y psicológica con su bebé, b) comparten y aceptan las emociones negativas generadoras de malestar de su hijo o hija pequeña, y c) buscan reducir o eliminar el estrés de su bebé, al tiempo que comparten emociones positivas, cuyo propósito es generar bienestar, satisfacción y disfrute mutuo (Posada \& Waters, 2014). A su vez, las madres que apoyan con cuidado sensible y comportamientos reparatorios aquellas descoordinaciones que se dan en las interacciones con el bebé - tal como se observó en este patrón de cuidado- favorecen en este la capacidad de afrontar los eventos estresores que ocurren en las rutinas de cuidado diario (Tronick $\&$ DiCorcia, 2015).

Por el contrario, en el patrón Regulatorio poco/no Contingente, las madres mostraban enorme dificultad para sintonizarse y armonizar su conducta con las señales comunicativas y necesidades de bebé. Igualmente, empleaban estrategias caracterizadas por la evitación de la proximidad y el contacto físico con el bebé, mientras este estaba estresado y llorando, lo cual se acompañaba de expectativas poco reales sobre a la capacidad del bebé para autorregularse. Según el relato de las madres, esta estrategia les permitía autorregularse y tranquilizarse cuando se sentían desbordadas emocionalmente por las 
emociones del bebé y el estrés generado por la crianza. Según Leerkes et al. (2004), basados en el modelo emociones y metas de crianza de Dix (1991), la madre se comporta según las reacciones emocionales que la ansiedad y el estrés del bebé le generen. En el caso que las emociones negativas del bebé le produzcan ansiedad o rabia, como ocurre en este patrón, ella tenderá a focalizarse en sus propias metas y necesidades, no tendrá en cuenta las necesidades y emociones del bebé, y se aislará de este con el propósito de evitar o finalizar la situación de estrés que percibe como aversiva.

Las estrategias propias del patrón Regulatorio poco/no Contingente empleadas por algunas madres involucraban expectativas de autorregulación del propio bebé sin el apoyo materno, comportamiento que va en dirección opuesta a la evidencia aportada por diversas investigaciones, que muestran que las experiencias emocionales intensas, tanto negativas como positivas, desbordan la capacidad del bebé para autorregularse, y por ello requiere de la co-regulación del cuidador que le guíe en este proceso (Cooper et al., 2007; Sroufe, 2016).

Este patrón, que se caracteriza por periodos prolongados de emociones negativas en el bebé y pocas reparaciones de los errores interactivos y faltas de sincronía en las interacciones en un periodo temprano del desarrollo, podría afectar la capacidad de afrontamiento del estrés en la vida cotidiana (Tronick \& DiCorcia, 2015).

Esta estrategia rompe el contexto diádico necesario para que la co-regulación emocional funcione como un sistema de regulación guiada (Sroufe et al., 2005). En este segundo patrón, las madres manifiestan cierta dificultad para acompañar los estados de activación fisiológica, afectiva y comportamental de su hijo de manera adecuada, aspecto que va en contravía de lo sugerido por los expertos (Calkins \& Hill, 2007; Feldman, 2012; Miller \& Commons, 2010), y que podría afectar negativamente la construcción de relaciones de apego seguro bebémadre (Sroufe et al., 2005, 2014). En el caso de los bebés irritables, se ha observado que en el primer año de vida, los bebés reducen su reactividad emocional (sus emociones negativas) ante la frustración, en función de la calidad del apego bebé-madre (Sherman et al., 2013). En el mismo sentido, Beebe et al. (2010) encontraron que las interacciones madre-bebé que son inconsistentes, y no contingentes a los cuatro meses, están asociadas al desarrollo de patrones de apego inseguro a los 12 meses.

Otro rasgo de este patrón es la baja capacidad de la madre para mostrar disponibilidad emocional y física ante la emocionalidad negativa del bebé, ocasionada porque la madre atribuye intencionalidades negativas al llanto y comportamiento de su hijo. De acuerdo al modelo de crianza de Dix (1991), aquellas madres que hacen atribuciones negativas $O$ una lectura distorsionada de las emociones y comportamientos del bebé, tienden a centrarse en sus propias necesidades y metas de crianza y, en consecuencia, no se focalizan ni responden a las necesidades de este, por lo que adoptan expectativas poco reales sobre la capacidad del bebé para autorregularse. Lo anterior evidencia no solo que la estrategia no es eficaz para coregular las emociones del bebé, sino también que la madre se estresa significativamente ante la situación. Estos resultados pondrían en evidencia lo que Cooper et al. (2007) y Leerkes et al. (2004) describen como una baja capacidad de empatía de los padres hacia los estados de sus hijos, que deriva en un aumento de la probabilidad de hacer atribuciones negativas a las señales y necesidades de los niños.

Al adoptar altas expectativas sobre la capacidad real del bebé para regularse por sí mismo, las madres con patrón poco/ no contingente también manifestaban escaso conocimiento sobre el desarrollo infantil, lo cual puede indicar que las que poseen poca escolaridad y falta de conocimiento sobre el desarrollo infantil tienden a tener creencias y expectativas muy bajas o muy altas sobre el comportamiento de sus hijos (Solís-Cámara \& Díaz Romero, 2006), lo que iría en detrimento de la calidad de la relación madre-bebé y de su proceso de desarrollo autorregulatorio. Pese a que el deseo es calmar el llanto y tranquilizar a su bebé, al hacer una interpretación negativa de sus comportamientos, tienen mayor dificultad 
para responder contingentemente sus señales, lo cual puede dificultar el logro de una de las metas del desarrollo que plantean Sroufe et al. (2005), referidas a la capacidad del bebé para expresar adecuadamente sus impulsos, deseos y sentimientos y aprender a autorregularse.

Ahora bien, ante la pregunta de este estudio, si el canto materno era empleado como estrategia regulatoria en situaciones de estrés, los resultados muestran que más de la mitad de las madres cantaban a sus bebés en distintos contextos y situaciones. No obstante, se pudo identificar que el canto materno tiene varias funciones que cualifican el cuidado y la relación madrebebé, entre ellas calmarlo ante la presencia de emocionalidad negativa, y por ello puede decirse que el canto de la madre sí cumple una función regulatoria. Este hallazgo corrobora y aporta nueva evidencia empírica sobre el canto materno en la crianza como estrategia de regulación emocional, tal como han encontrado otros investigadores (Altmann de Litvan et al., 2001; Bauer et al., 2007; Baker \& Mackinlay, 2006; Bargiel, 2004; LEtoile, 2006; Trehub et al., 2015; Vrushali \& Shefali, 2011).

Por otra parte, también se halló que entre los géneros musicales empleados, primaban las canciones infantiles animadas y bailables populares, y que se hacía poco uso de las canciones de cuna tradicionales. A pesar de que se ha encontrado en algunas investigaciones que el uso de la canción de cuna tradicional se mantiene y ha trascendido histórica y culturalmente a través de la oralidad por generaciones (Trehub \& Trainor, 1998), otros autores señalan que las canciones de cunas españolas de la América Hispana u otras de origen Amerindio no se conservan por completo (Fernández, 2005; Tejero, 2002). Lo anterior parecería indicar que las prácticas de crianza y cuidado, entre estas, el canto a los bebés, se contextualizan en las experiencias cotidianas y socioculturales de sus entornos. Así mismo, en concordancia con Trehub et al. (2015), los niños se regulan emocionalmente mejor cuando las madres les cantan canciones animadas multimodales en las situaciones estresantes durante el día, que con las canciones de cuna, las cuales, al ser más suaves, parecen reservarse con mayor frecuencia para la hora de dormir.

Los hallazgos obtenidos son prometedores, aunque preliminares, por tanto, no generalizables, en cuanto que permitieron identificar dos patrones comportamentales maternos en las prácticas de cuidado con bebés en contextos de la vida cotidiana, en población con vulnerabilidad socioeconómica. Uno de los patrones de cuidado caracterizados podría considerarse favorecedor del desarrollo socioemocional y del bienestar infantil. Sin embargo, el otro patrón permite visualizar cómo los estresores cotidianos en los ambientes de cuidado temprano, junto con otras variables de las distintas ecologías micro y macro, en las que se encuentran inmersas las familias, pueden dificultar la relación madre-bebé, con prácticas no favorecedoras del desarrollo socioemocional. De igual manera, se halló evidencia de que la canción materna cumplía varias funciones en el cuidado infantil, y una de ellas era la regulación emocional de los bebés cuando estaban estresados en las rutinas de cuidado diario.

Un aspecto a resaltar de este estudio es el uso de medidas observacionales con video y de reporte parental, con cuestionarios de pregunta abierta, así como sistemas de codificación de la información con software digital, pues permiten identificar patrones interactivos madre-bebé y evaluar aspectos cuantitativos y cualitativos de dichas interacciones, en términos de sentimientos, necesidades y creencias que subyacen a las prácticas de crianza y cuidado infantil, las cuales han sido empleadas por algunos equipos de investigadores de los campos de conocimiento de familia, infancia y regulación emocional (Hsu \& Fogel, 2001; Lucassen et al., 2015; Pratt et al., 2015).

\section{Limitaciones y proyecciones del estudio}

Una de las limitaciones del estudio es el tamaño y la procedencia homogénea de la muestra (contexto de pobreza), lo cual exige, para futuros estudios, trabajar con muestras que 
tengan mayor representatividad estadística y de diferentes niveles socioeconómicos y educativos. Así mismo, para investigar sobre los procesos co-regulatorios de los bebés, sería recomendable utilizar el enfoque sistémico de la familia (Feldman, 2012), ya que permite explorar el papel que juega el padre y otras figuras familiares cercanas en dichos procesos, si se considera que en los contextos familiares de cuidado infantil contemporáneos, no solo la madre juega un rol central en la crianza de los niños pequeños, sino también otras figuras familiares, como el padre, que en las últimas décadas ha mostrado involucramiento en la crianza y cuidado temprano cada vez mayor (Shorey, \& Ang, 2019; Shorey, Ang, Goh, \& Gandhi, 2019).

También es relevante diseñar investigaciones para poder identificar diferencias en las estrategias regulatorias y en el cuidado temprano entre padres y madres, así como las contribuciones únicas del padre (Baker, Kainz, \& Reynolds, 2018; Feldman, Gordon et al., 2010; Feldman, 2012; Gärtner, Vetter, Schäferling, Reuner, \& Hertel, 2018; Lucassen et al., 2015), para comprender qué otros procesos de la cocrianza inciden en el involucramiento parental (como la autoeficacia), y cómo estos se relacionan con el desarrollo socioemocional, específicamente la capacidad autorregulatoria de los niños. Aunque en la muestra, las madres provenían de diferentes configuraciones familiares como las nucleares, en unión legal o en convivencia, monoparental femenina y extensa con parientes de la madre, desde la perspectiva de familia, es relevante considerar estudios comparativos con muestras de configuraciones familiares diversas, que cada vez son más en el país y en Latinoamérica (Ullmann, Maldonado Valera, \& Rico, 2014).

A partir de los hallazgos de este estudio, se podría abrir un campo de investigación para comprender las relaciones entre las estrategias regulatorias maternas y paternas - considerando procesos como la co-regulación y el contacto físico en el primer año de vida, y constructos de la teoría del apego, como son la sensibilidad (Posada \& Waters, 2014) y la capacidad de mentalización (Fonagy, Gergely, Jurist, \& Target,
2018) - y, a su vez, entender cómo se articulan las estrategias regulatorias maternas y paternas con la construcción de las relaciones de apego temprana bebé-madre o bebé-padre.

En esa medida se enfatiza la relevancia de realizar estudios de procesos familiares, prácticas de crianza y desarrollo infantil desde las etapas más tempranas de las relaciones madre/padrebebé, por sus implicaciones para políticas públicas y programas de prevención y promoción de la calidad de los ambientes de cuidado en la primera infancia, especialmente en los contextos psicosocialmente vulnerables.

\section{Agradecimientos}

Esta publicación es derivada de un proyecto de investigación mayor, multi-sitio, "Canción de cuna como una de las estrategias de regulación emocional y sus relaciones con la sensibilidad materna y las representaciones mentales maternas del apego y los precursores del apego infantil: En el contexto Latinoamericano". Muestra Colombia. Fue diseñado conjuntamente entre el equipo investigador de la ONG, ATI Atención y Desarrollo a la Temprana Infanciadel Uruguay, bajo la coordinación de Marina Altmann, PhD., y el equipo de investigación de la Línea de Relaciones Vinculares del Grupo Desarrollo, Afectividad y Cognición de la Pontificia Universidad Javeriana. Cofinanciado por la Pontificia Universidad Javeriana y la Fundación para la Promoción de la Investigación y la Tecnología. Banco de la República, Proyecto N. ${ }^{\circ}$ 3.209. 3.

\section{Referencias}

Ainsworth, M. D. S., Bell, S. M., \& Stayton, D. J. (1974). Infant-Mother Attachment and Social Development. En M. P. Richards (Ed.), The Introduction of the Child into a Social World (pp. 99-135). Londres: Cambridge University Press.

Altmann de Litvan, M., Weigensberg de Perkal, A., González, E., Bauer, M., Angulo, B., Brovetto, E., ...Vieira, M. (2001). 
Arrullos, ritmos y sincronías en la relación madre-bebé. Revista Iberoamericana de Psicomotricidad y Técnicas Corporales, 1, 49-62.

Baker, C. E., Kainz, K. L., \& Reynolds, E. R. (2018). Family Poverty, Family Processes and Children's Preschool. Journal of Child and Family Studies, 27(4), 1242-1251. https ://doi.org/10.1007/s10826-017-0947-6

Baker, F., \& Mackinlay, E. (2006). Sing, soothe and sleep: A lullaby education programme for first-time mothers. British Journal of Music Education, 23(2), 147-160. https://do i.org/10.1017/S0265051706006899

Bargiel, M. (2004). Lullabies and play songs: Theoretical considerations for an early attachment music therapy intervention through parental singing for developmentally at-risk infants. Voices: A World Forum for Music Therapy, 4(1). https: //doi.org/10.15845/voices.v4i1.149

Bauer, M., González. E., Sassón, E., Weigensberg, A., Corti, A., \& Altmann de Litvan, M. (2007). Vínculo temprano y promoción de resiliencia. Una experiencia de trabajo. En Autores (Eds.), Resiliencia y vida cotidiana (pp. 269-294). Montevideo: Psicolibros.

Beebe, B., Jaffe, J., Markese, S., Buck, K., Chen, H., Cohen, P., ...Feldstein, S. (2010). The origins of 12 -month attachment: A microanalysis of 4-month motherinfant interaction. Attachment $\mathcal{E}$ Human Development, 12 (1-2), 3-141. https://doi.or $\mathrm{g} / 10.1080 / 14616730903338985$

Bowlby, J. (1993). El Vínculo Afectivo. Barcelona: Paidós.

Calkins, S. D., \& Hill, A. (2007). Caregiver influences on emerging emotion regulation: Biological and environmental transactions in early development. En J. J. Gross (Ed.), Handbook of emotion regulation (pp. 229-248). Nueva York, NY: Guilford Press.

Causadias, J. M., Salvatore, J. E., \& Sroufe, L. A. (2012). Early patterns of selfregulation as risk and promotive factors in development: A longitudinal study from childhood to adulthood in a high-risk sample. International Journal of Behavioral
Development, 36(4), 293-302. https://doi.or g/10.1177/0165025412444076

Cicchetti, D., \& Valentino, K. (2006). An ecological-transactional perspective on child maltreatment: Failure of the average expectable environment and its influence on child development. En D. Cicchetti \& R. J. Cohen (Eds.), Developmental psychopathology. Risk, disorders, and adaptation (pp. 129-201). Hoboken, NJ: John Wiley \& Sons.

Cooper, G., Hoffman, K., Powell, B., \& Marvin, R. (2007). The circle of security intervention: Differential diagnosis and differential treatment. En L. J. Berlin, Y. Ziv, L. Amaya-Jackson, \& M. Greenberg (Eds.), Enhancing Early Attachments: Theory, research, intervention, and policy (pp. 127-151). Nueva York, NY: The Guilford Press.

Coppola, G., Ponzetti, S., Aureli, T., \& Vaughn, B. E. (2016). Patterns of emotion regulation at two years of age: associations with mothers' attachment in a fear eliciting situation. Attachment \& human development, 18(1), 16-32. https://doi.org/ 10.1080/14616734.2015.1109676

Crespo, L. M., Trentacosta, C. J., Aikins, D., \& Wargo-Aikins, J. (2017). Maternal emotion regulation and children's behavior problems: The mediating role of child emotion regulation. Journal of Child and Family Studies, 26(10), 2797-2809. https://d oi.org/10.1007/s10826-017-0791-8

De Rubeis, S., \& Granic, I. (2012). Understanding treatment effectiveness for aggressive youth: The importance of regulation in mother-child interactions. Journal of Family Psychology, 26(1), 66-75. h ttps://doi.org/10.1037/a0026837

Departamento Administrativo de Planeación Distrital. (2005). La estratificación en Bogotá D.C. y estudios relacionados 1983-2004. Bogotá: Alcaldía Mayor.

Diener, M. L., Mangelsdorf, S. C. McHale, J. L., \& Frosch, C. (2002). Infants' behavioral strategies for emotional regulation with fathers and mothers: Associations with 
emotional expressions and attachment quality. Infancy, 3(2), 153-174. https://doi.o $\mathrm{rg} / 10.1207 / \mathrm{S} 15327078 \mathrm{IN} 03023$

Dix, T. (1991). The affective organization of parenting: Adaptive and maladaptive processes. Psychological Bulletin, 110(1), 3-25.

Feldman, R. (2012). Bio-behavioral synchrony: A model for integrating biological and microsocial behavioral processes in the study of parenting. Parenting: Science and Practice, 12(2-3), 154-164. https://doi.org/1 $0.1080 / 15295192.2012 .683342$

Feldman, R., \& Eidelman, A. I. (2009). Biological and environmental initial conditions shape the trajectories cognitive and socialemotional across the first five years of life. Developmental Science, 12(1), 194-200.

Feldman, R., Gordon, I., Schneiderman, I., Weisman, O., \& Zagoory-Sharon, O. (2010). Natural variations in maternal and paternal care are associated with systematic changes in oxytocin following parent-infant contact. Psychoneuroendocrinology, 35(8), 1133-1141. https://doi.org/10.1016/j.psyne uen.2010.01.013

Feldman, R., Singer, M., \& Zagoory, O. (2010). Touch attenuates infants' physiological reactivity to stress. Developmental Science, 13(2), 271-278. https://doi.org/10.1111/j.1 467-7687.2009.00890.x

Fernández, A. (2005) Canción de cuna: Arrullo o Desvelo. Anales de Antropología, 39(2), 189-212.

Fonagy, P., Gergely, G., \& Jurist, E. L. (2018). Affect Regulation, Mentalization and the Development of the Self. London: Routledge, https://doi.org/10.4324/9780429471643

Gärtner, K. A., Vetter, V. C., Schäferling, M., Reuner, G., \& Hertel, S. (2018). Inhibitory control in toodlerhood - the role of parental co-regulation and self-efficacy beliefs. Metacognition and Learning, 13(3), 241-264. https://doi.org/10.1007/s11409-0 18-9184-7

González Ramírez, M. C. (2011). Relación entre la sensibilidad materna, calidad del apego bebé-mamá y tipos de estrategias maternas e infantiles de regulación emocional (Informe final de investigación. Jóvenes Investigadores e Innovadores-Colciencias, no publicado). Convocatoria 5252011.

Hackman, D. A., Gallop, R., Evans, G. W., \& Farah, M. J. (2015). Socioeconomic status and executive function: Developmental trajectories and mediation. Developmental Science, 18(5), 686-702. https://doi.org/10. 1111/desc. 12246

Hernández, R., Fernández, C., \& Baptista, P. (2010) Metodología de la investigación. Ciudad de México: McGraw Hill.

Hsu, H., \& Fogel, A. (2001). Infant Vocal Development in a Dynamic Mother-Infant Communication System. Infancy, 2(1), 87-109.

Idoyaga, A. (2008). Del Arrorró a la calidez del sueño. Las canciones de cuna y el mestizaje cultural en el área de Cuyo. Huellas, Búsquedas en Artes y Diseño, 6, 155-163.

Im-Bolter, N., Anam, M., \& Cohen, N. J. (2015). Mother-child synchrony and child problem behavior. Journal of Child and Family Studies, 24(7), 1876-1885. https://doi.org/10.1007/s 10826-014-9989-1

Jean, A. D. L., Stack, D. M., \& Arnold, S. (2014). Investigating maternal touch and infants' self- regulatory behaviours during a modified face-to-face still-face with touch procedure. Infant and Child Development, 23(6), 557-574. https://doi.org/10.1002/icd .1870

King, L. S., Humphreys, K. L., \& Gotlib, I. H. (2019). The neglectenrichment continuum: Characterizing variation in early caregiving environments. Developmental Review, 51, 109-122. https:// doi.org/10.1016/j.dr.2019.01.001

LEtoile, S. (2006). Infant-directed singing: A theory for clinical intervention. Music Therapy Perspectives, 24(1), 22-29. https://d oi.org/10.1093/mtp/24.1.22

Leerkes, E. M., Blankson, A. N., \& O'Brien, M. (2009). Differential Effects of Maternal Sensitivity to Infant Distress and NonDistress on Social-Emotional Functioning. 
Child Development, 80(3), 762-775. https:// doi.org/10.1111/j.1467-8624.2009.01296.x

Leerkes, E. M., Crockenberg. S. C., \& Burrous, C. E. (2004). Identifying components of maternal sensitivity to infant distress. The role of maternal emotional competencies. Parenting, Science, and Practice, 4(1), 1-23. https://doi.org/10.1207/s15327922pa r0401_1

Lipina, S. J., \& Segretin, M. S. (2015). 6000 días más: evidencia neurocientífica acerca del impacto de la pobreza infantil. Psicología Educativa, 21 (2), 107-116. https://doi.org/1 0.1016/j.pse.2015.08.003

Little, C., \& Carter, A. S. (2005). Negative emotional reactivity and regulation in 12 months-olds following emotional challenge: Contribution of maternal-infant emotional availability in a low-income sample. Infant Mental Health Journal, 26(4), 354-368. https://doi.org/10.1002/imhj.200 55

Lucassen, N., Kok, R., Bakermans-Kranenburg, M. J., Van Ijzendoorn, M. H., Jaddoe, V. W. V., Hofman, A., ...Tiemeir, H. (2015). Executive functions in early childhood: The role of maternal and paternal parenting practices. British Journal of Developmental Psychology, 33(4), 489-505. https://doi.org/ 10.1111/bjdp.12112

Miller, P., \& Commons, M. (2010). The Benefits of Attachment Parenting for Infants and Children: A Behavioral Developmental View. Behavioral Development Bulletin, 16(1), 1-14. https://doi.org/10.1037/h0100 514

Naciones Unidas. (2015). Objetivos de desarrollo sostenible (ODS). Recuperado de https://www.undp.org/content/undp/es/ home/sustainable-development-goals.html

Nelson, C. A., Furtado, E., Fox, N. A., \& Zeanah, C. (2009). The deprived human brain. American Scientist, 97(3), 222-229. https:// doi.org/10.1511/2009.78.222

Perry, R. E., Blair, C., \& Sullivan, R. M. (2017). Neurobiology of infant attachment: attachment despite adversity and parental programming of emotionality. Current opinion in psychology, 17, 1-6. https://doi.org /10.1016/j.copsyc.2017.04.022

Posada, G., \& Waters, E. (2014). El sistema de comportamiento de cuidado: Sensibilidad y apoyo de base segura. En B. Torres, J. Causadias, \& G. Posada (Eds.), La teoría del apego: Investigación y aplicaciones clínicas (pp. 75-97). Madrid: Psimática Editorial.

Pratt, M., Singer, M., Kanat-Maymon, Y., \& Feldman, R. (2015). Infant negative reactivity defines the effects of parent-child synchrony on physiological and behavioral regulation of social stress. Development and psychopathology, 27(4), 1191-1204. https://d oi.org/10.1017/S0954579415000760

Qu, J., Leerkes, E. M., \& King, E. K. (2016). Preschoolers' distress and regulatory behaviors vary as a function of infant-mother attachment security. Infant Behavior and Development, 44, 144-147. htt ps://doi.org/10.1016/j.infbeh.2016.06.008

Retana Franco, B. E., \& Sánchez-Aragón, R. (2010). Rastreando en el pasado...las formas de regular la felicidad, la tristeza, el amor, el enojo y el miedo. Universitas Psychologica, 9(1), 179-197. https://doi.org/ 10.11144/Javeriana.upsy9-1.rpfr

Riva Crugnola, C., Tambelli, R., Spinelli, M., Gazzotti, S., Caprin, C., \& Albizzati, A. (2011). Attachment patterns and emotion regulation strategies in the second year. Infant Behavior \&ु Development, 34(1), 136-151. https://doi.org/10.1016/j.infbeh.2 010.11 .002

Satchwell-Hirst, M. (2018). Neuroscience and Emotional Development. The basics Explained. En D. Colley \& P. Cooper (Eds.), Attachment and Emotional Development in the Classroom. Theory and Practice (pp. 49-73). Londres: Jessica Kingsley Publishers.

Shenfield, T., Trehub, S. E., \& Nakata, T. (2003). Maternal singing modulates infant arousal. Psychology of Music, 31 (4), 365-375. https:/ /doi.org/10.1177/03057356030314002

Sherman, L. J., Stupica, B., Dykas, M. J., Ramos-Marcuse, F., \& Cassidy, J. (2013). The development of negative reactivity 
in irritable newborns as a function of attachment. Infant Behavior Eु Development, 36(1), 139-146. https://doi.org/10.1016/j.in fbeh.2012.11.004

Shorey, S., \& Ang, L. (2019). Experiences, needs, and perceptions of paternal involvement during the first year after their infants' birth: A meta-synthesis. Plos One, 14(1), 1-21. htt ps://doi.org/10.1371/journal.pone.0210388

Shorey, S., Ang, L., Goh, E. C. L., \& Gandhi, M. (2019). Factors influencing paternal involvement during infancy: A prospective longitudinal study. Journal of Advanced Nursing, 75(2), 357-367. https://doi.org/10. $1111 /$ jan. 13848

Solís-Cámara, P., \& Díaz Romero, M. (2006). Efectos de un programa de crianza para mamás y papás de niños pequeños: La importancia del nivel educativo de los padres. Revista Infancia, Adolescencia y Familia, 1(1), 161-176.

Sroufe, A., Szteren, L., \& Causadias, J. (2014). El apego como sistema dinámico: fundamentos de la teoría del apego. En B. Torres Gómez de Cádiz, J. M. Causadias, \& G. Posada (Eds.), La teori\# a del apego: Investigacio\#n y aplicaciones cli\#nicas (pp. 27-39). Madrid: Editorial Psimática.

Sroufe, L. A. (2016). The place of attachment in development. En J. Cassidy, \& P. R. Shaver (Eds.), Handbook of Attachment. Theory, Research, and Clinical Applications (pp. 997-1011). Nueva York, NY: The Guilford Press.

Sroufe, L. A., Egeland, B., Carlson, E. A., \& Collins, W. A. (2005). The Development of the person: The Minnesota study of risk and adaptation from birth to adulthood. Nueva York, NY: Guilford Press.

Tejero, E. (2002). La canción de cuna y su función de catarsis en la mujer. Didáctica (Lengua y Literatura), 14, 211-232.

Thomas, J. C., Letourneau, N., Campbell, T. S., Tomfohr-Madsen, L., \& Giesbrecht, G. F. (2017). Developmental origins of infant emotion regulation: Mediation by temperamental negativity and moderation by maternal sensitivity. Developmental
Psychology, 53(4), 611-628. https://doi.org/ 10.1037/dev0000279

Trehub, S. E., \& Trainor, L. (1998). Singing to infants: Lullabies and playsongs. Advances in Infancy Research, 12, 43-78.

Trehub, S., Ghazban, N., \& Corbeil, M. (2015). Music affect regulation in infancy. Annals of the New York Academy of Sciences, 1337(1), 186-192. https://doi.org/10.1111/nyas.1262 2

Tronick, E., \& DiCorcia, J. A. (2015). The everyday stress resilience hypothesis: A reparatory sensitivity and the development of coping and resilience. Children Australia, 40(2), 124-138. https://doi.org/10.1017/ch a.2015.11

Ullmann, H., Maldonado Valera, C., \& Rico, M. N. (2014). La evolución de las estructuras familiares en América Latina, 1990-2010. Los retos de la pobreza, la vulnerabilidad y el cuidado. Santiago: Naciones Unidas.

Unicef. (2009). Estado mundial de la infancia. Conmemoración de los 20 años de la Convención sobre los derechos del niño (Edición Especial). Nueva York, NY: Naciones Unidas. Recuperado de https://www.unicef.org/spanish/publicat ions/files/SOWC_Spec._Ed._CRC_Main_ Report_SP_1201009.pdf

Vrushali, P., \& Shefali, M. (2011). Psychological effect of lullabies in child development. Indian Journal of Positive Psychology, 8(4), 677-680.

Winston, R., \& Chicot, R. (2016). The importance of early bonding on the longterm mental health and resilience of children. London JournaL of Primary Care, 8(1), 12-14. https://doi.org/10.1080/17571 472.2015 .1133012

\section{Notas}

* Artículo de investigación. 